adequate for the measurement of the elements of common biological interest and, although the trials are not yet complete, an overall accuracy of $I$ to $2 \%$ is anticipated. The completed in- strument will be fully automated with automatic dilution of the sample and print-out of the analytical results.

\title{
REFERENCES
}

Dawson, J. B., and Heaton, F. W. (r96r): The Determination of Magnesium in Biological Materials by Atomic Absorption Spectrophotometry. Biochem. Y., 80, 99.

Willis, J. B. (1963): Methods of Biochemical Analysis. D. Glick (Ed.). New York : Interscience Publ., pp. I-67.

\section{X-RAY IMAGE DETECTION BY ELECTRONIC METHODS}

\author{
G. A. $\mathrm{HAY}_{\mathrm{A}}$
}

In diagnostic radiology, the excellence of the conventional radiographic screen-film combination as a means of detecting and displaying the X-ray pattern (the radiation image) is qualified by its inability to show movement. The technique of fluoroscopy, in which the light image on the fluorescent screen is viewed by the dark-adapted but otherwise unaided eye, has long been employed in an attempt to overcome this disadvantage. Unfortunately, because of restricted optical aperture and low retinal efficiency, the eye responds to only a very small fraction of the light emitted by the screen, and only about one-fifth of the information inherent in the screen image is transmitted to the brain. This inefficiency of information transfer also renders it necessary in clinical work to use dose-rates which are higher than appear at present to be desirable. These fundamental deficiencies, together with the inconvenience of the darkened room which can be a great handicap in complex techniques such as cardiac catheterization, seriously limit the clinical applications of fluoroscopy.

Many attempts have therefore been made to improve the collection of light and therefore of information from the fluoroscopic screen. The first successful device, called the X-ray image intensifier tube (Coltman, 1948), had a high efficiency of light collection, but also a number of disadvantages. The field size was restricted to about $4 \frac{1}{2}$ inches in diameter, and the quality of the image was greatly affected by contrast loss due to various internal factors. Because it was necessary to view the image through a microscope, the radiologist's movements were seriously restricted.

In 1957, after considerable theoretical study, work on a new type of X-ray image detector (first described by Morgan and Sturm, I95I) was undertaken in this laboratory, assisted by a generous grant from the Nuffield Foundation. In this image detector the fluoroscopic screen is viewed by a television camera incorporating a large aperture lens and a very sensitive television camera tube, the image orthicon. After amplification in the electronic circuits the output image is displayed on the screen of a conventional television receiver. Initial experiments were first carried out with standard television equipment, and subjective assessments were made of the informationgathering properties of the system (Hay, 1958; I960). In the present experimental system, a $12 \frac{1}{2}$ in. diameter fluoroscopic screen is used in conjunction with an $\mathrm{f} / \mathrm{o.68}$ mirror optical system (Bouwers, 1950) and a special $4 \frac{1}{2}$ in. image orthicon tube (Banks, 1958). In this system, although considerable light is lost between screen and photocathode, the light transfer is about one hundred times that of the eye and little information should in principle be lost. Subjective measurements of information transfer (Hay, 1962) show that at conventional dose-rates the improvement over fluoroscopy is about five times; conversely for image quality approximating to conventional fluoroscopy the dose-rate may be reduced generally to about one-twentieth that of fluoroscopic levels.

This image detector has been used in a variety of clinical procedures, and as might be expected it displays all the advantages generally associated with television techniques. The radiologist and his collaborators can work in subdued artificial light and complex techniques such as cardiac catheterization are greatly facilitated, and are often so accelerated that a substantial dose reduction to the patient results from reduced operating time as well as from the lower dose-rate. The facilities available for the electronic control of contrast and other image parameters are useful; for example, the inequalities of X-ray transmission seen in the region of the diaphragm can be compensated so that an image of even overall illumination is obtained. Radiological demonstrations are possible to large audiences (the technique is being used in this University in the teaching of human physiology to medical students) and the moving image may be recorded either on cine-film via the television screen or on magnetic tape.

Television systems of various types are now in widespread use in radiology. There is a commercial version of the system described above, and the 
early X-ray image intensifier tube has in recent years been substantially improved and its output is now commonly observed via a small television camera. There is no doubt that in time conventional fluoroscopic techniques will disappear and at present the only alternative is the television method. Before this can occur, great technological advances will be necessary. For example, one development which is being pursued in this laboratory makes use of a highly sensitive type of image orthicon (with a storage target of magnesium oxide in place of glass). Experiments (Hay and Clarke, 1962) show that the use of this tube should enable clinical dose levels to be reduced by a further factor of ten. Such a low dose-rate however may, as indicated below, imply an unacceptable limitation on the information present in the image.

During the technological developments described above, two broad fundamental problems have been encountered. First, it is important to be able to estimate the quality or information content of an image at any desired X-ray dose-rate, so that the minimum patient dose consistent with adequate information transfer can be selected. The information inherent in any radiation image (formed by X-rays, visible light, etc.) is a function of the number of quanta per unit area per unit time, and also of the distribution of quantum energies in the image. In its passage through any practical image-detecting system, the information content of course deteriorates, the loss resulting both from transfer inefficiencies and from technical deficiencies such as contrast loss. These phenomena are governed in some way by statistical laws but relatively little is understood of how these laws⿳⺈冂䒑 should be applied. Some attempt is being mades in this laboratory to pursue such enquiries; for example, the information content of X-rayc. images at different quantum energies is being studied, and the perceptibility of random images synthesised electronically on a television screen is? being assessed.

The second problem is that of measuring the

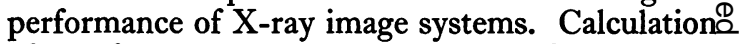
of performance, even in terms of measured parameters, is so difficult that for practicalpurposes, e.g. in the hospital department, $a$ direct subjective assessment is at present essential. $\vec{\omega}$ Such subjective measurements have been known for many years, but an improved method (Hay? 1964) has been developed here which not only? allows comparison of system performances but which also attempts to relate performance to theory. This method has been applied to the assessment of our own image detectors, and to a number of other $\vec{f}$ systems in this country and in Europe and America.o It is almost equally important that the results of ${ }^{5}$ such a test should be capable of simple expression, $\vec{z}$ preferably as a single image parameter. Formalo methods using an extension of Shannon's inform-⿳⺈ ation theory are difficult to apply and bear littlepractical relation to the radiological problem. I stead, a simpler concept, the information index, whish combines contrast sensitivity and detail resolution has been suggested (Hay, I958; 1960) as beings more directly related to the special requirementso of radiation image assessment. Much theoreticak़ work remains to be done in this field and these problems are now being studied.

\section{REFERENCES}

Banks, G. B. (1958): Television Pick-up Tubes for X-ray Screen Intensification, Brit. F. Radiol., 31, 6 I9.

Bouwers, A. (1950): 'Achievements in Optics'. Amsterdam: Elsevier.

Coltman, J. W. (1948): Fluoroscopic Image Brightening by Electronic Means, Radiology, 51, 359.

HAY, G. A. (r958): Quantitative Aspects of Television Techniques in Diagnostic Radiology, Brit. $\mathcal{F}$. Radiol., 3r, 6r r. (1960): X-ray Image Intensification Using Optical Television Methods. In 'Advances in Electronics and Electron Physics', vol. 12, 263. New York: Academic Press. (1962): The Image Orthicon in Diagnostic Radiology. In 'Advances in Electronics and Electron Physics', vol. I6을 and Clarke, O. F. (1962): The Image Orthicon with Magnesium Oxide Target in X-ray. Image Detectiono
Brit. F. Radiol., 35, 2 19.

(r964): A Physical Assessment of the Cinelix Electro-optical Image Intensifier in Television Fluoroscopy
Radiology, May, r964 (in the press). Morgan, R. H., and Sturm, R. E. (195I): The Johns Hopkins Fluoroscopic Screen Intensifier, Ibid., 57, 556. 